\title{
Preparation and Characterization of a Calcium Carbonate Aerogel
}

\author{
Johann Plank, ${ }^{1}$ Heinz Hoffmann, ${ }^{2}$ Joachim Schölkopf, ${ }^{3}$ Wolfgang Seidl, ${ }^{1}$ \\ Ingo Zeitler, ${ }^{2}$ and Zheng Zhang ${ }^{3}$
}

${ }^{1}$ Chair for Construction Chemicals, Technische Universität München, Lichtenbergstraße 4, 85747 Garching bei München, Germany

${ }^{2}$ Bayreuth Center for Colloids and Interfaces, Universität Bayreuth, Universitätsstraße 30, 95440 Bayreuth, Germany

${ }^{3}$ Omya Development AG, Baslerstraße 42, P.O. Box 335, 4665 Oftringen, Switzerland

Correspondence should be addressed to Johann Plank, sekretariat@bauchemie.ch.tum.de

Received 22 October 2008; Accepted 2 April 2009

Recommended by Manish U. Chhowalla

We report on a facile method for the preparation of a calcium carbonate aerogel consisting of aggregated secondary vaterite particles with an approximate average diameter of $50 \mathrm{~nm}$. It was synthesized via a sol-gel process by reacting calcium oxide with carbon dioxide in methanol and subsequent supercritical drying of the alcogel with carbon dioxide. The resulting monolith was opaque, brittle and had overall dimensions of $6 \times 2 \times 1 \mathrm{~cm}$. It was characterized by X-ray powder diffraction, nitrogen adsorption method (BET), and scanning electron microscopy.

Copyright (C) 2009 Johann Plank et al. This is an open access article distributed under the Creative Commons Attribution License, which permits unrestricted use, distribution, and reproduction in any medium, provided the original work is properly cited.

\section{Introduction}

The preparation of an aerogel was first described by Kistler in the 1930s. He synthesized a silica-based aerogel via a wet chemical sol-gel process and subsequent supercritical solvent extraction $[1,2]$. The aerogel showed unique material properties such as high optical transparency or opacity, heat insulation capability, and high absorption capacity $[3,4]$. These properties led to the use of silica aerogels in ion exchange materials, adsorbents, semipermeable membranes, pharmaceuticals, cosmetics, optical and acoustical devices, coating materials, and heat insulation applications for buildings [5-7]. Since then, various types of aerogels based on carbon [8], alumina $[9,10]$, transition metal oxides [11], or main-group metal oxides [12] were synthesized.

In 2007, Horga et al. reported on the preparation of a calcium carbonate aerogel for the first time [13]. Their process involves the ionotropic gelling of alginate in a calcium salt solution, followed by an exchange of the solvent water against ethanol, and supercritical drying of the calcium alginate alcogel. Calcination of the calcium alginate aerogel finally leads to a calcium carbonate aerogel. Obviously, this process requires many consecutive steps. In this communication we report on a more facile way to prepare a calcium carbonate aerogel resulting from the condensation of vaterite nanoparticles. These were prepared by controlled hydrolysis of calciumdi(methylcarbonate) which was obtained by the reaction of carbon dioxide with calcium oxide in absolute methanol. The resulting $\mathrm{CaCO}_{3}$ alcogel was subjected to supercritical drying with $\mathrm{CO}_{2}$ to produce the $\mathrm{CaCO}_{3}$ aerogel.

\section{Experimental Section}

2.1. Preparation of $\mathrm{CaCO}_{3}$ Sol. Buzagh's method was used to prepare the $\mathrm{CaCO}_{3}$ sol [14]. Thus, $54.0 \mathrm{~g}$ (0.963 mol) calcium oxide (calcined for $24 \mathrm{~h}$ at $950^{\circ} \mathrm{C}$ ) were suspended in $800 \mathrm{~mL}$ absolute methanol (dried and stored over molecular sieve, $3 \mathrm{~nm}$ ) and heated to $40^{\circ} \mathrm{C}$. After 90-minute stirring, carbon dioxide was bubbled through the reaction vessel for one hour at a flow rate of $1 \mathrm{~L} / \mathrm{min}$. The carbon dioxide was fed into the reaction vessel through a metal tube close to a stirrer $(1000 \mathrm{rpm})$ to ensure a fast reaction in the methanolic suspension. When all the calcium oxide was dissolved and converted into a sol, the flow of carbon dioxide was stopped. The resulting sol contained nanoparticles with particle sizes ranging from 5 to $20 \mathrm{~nm}$ (dynamic light scattering measurements, Zetasizer Nano-ZS, Malvern Instruments Ltd.). 


$$
\begin{aligned}
& \mathrm{CaO}+2 \mathrm{CH}_{3} \mathrm{OH} \\
& \begin{array}{ll}
\mathrm{CaO}+2 \mathrm{H}_{2} \mathrm{O} & \rightleftarrows \mathrm{Ca}(\mathrm{OH})_{2} \\
\mathrm{Ca}(\mathrm{OH})_{2}+2 \mathrm{CH}_{3} \mathrm{OH} & \rightleftarrows \mathrm{Ca}\left(\mathrm{OCH}_{3}\right)_{2}+2 \mathrm{H}_{2} \mathrm{O}
\end{array} \\
& \rightleftarrows \mathrm{Ca}\left(\mathrm{OCH}_{3}\right)_{2}+\mathrm{H}_{2} \mathrm{O} \\
& \mathrm{Ca}\left(\mathrm{OCH}_{3}\right)_{2}+2 \mathrm{CO}_{2} \longrightarrow \mathrm{Ca}\left(\mathrm{OCOOCH}_{3}\right)_{2} \\
& \mathrm{Ca}\left(\mathrm{OCOOCH}_{3}\right)_{2}+\mathrm{H}_{2} \mathrm{O} \longrightarrow \mathrm{CaCO}_{3}+2 \mathrm{CH}_{3} \mathrm{OH}+\mathrm{CO}_{2} \text { (III) }
\end{aligned}
$$

Scheme 1: Reaction steps involved in the formation of the $\mathrm{CaCO}_{3}$ sol from calcium oxide and gaseous carbon dioxide in methanol.

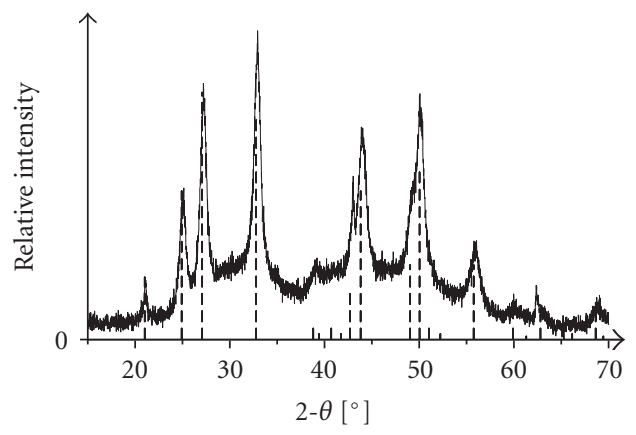

Figure 1: X-ray powder diffraction pattern of the $\mathrm{CaCO}_{3}$ aerogel. Vertical dotted lines correspond to JCPDS entry 33-0268 (vaterite).

The particle size distribution curve showed a maximum at approximately $11 \mathrm{~nm}$.

2.2. Sol-Gel Conversion. The sol turns into a translucent alcogel when stored for a short time ( $<1$ hour). The gelation time depends on the amount of water present in the reaction vessel and the temperature. When more water was present during the synthesis (e.g., because of not using absolute methanol), gelation already took place in the reaction vessel. Higher temperature also leads to faster sol-gel conversion. The alcogels obtained were stable for days when stored under refrigeration. In air and at room temperature, they dried to an opaque powder which, when being freshly prepared, consisted of pure vaterite particles.

2.3. Preparation of $\mathrm{CaCO}_{3}$ Aerogel. A part of the calcium carbonate alcogel was subjected to supercritical drying with carbon dioxide. The supercritical drying was carried out in a $1 \mathrm{~L}$ pressure autoclave (Parr Company, Germany). Approximately $18 \mathrm{~g}$ of alcogel were placed in the autoclave, and absolute methanol was added until the gel was completely immersed in the solvent. The autoclave was then sealed, pressurized slowly with carbon dioxide to 6.0 MPa and brought to a temperature of $281 \mathrm{~K}$. When equilibrium was achieved between the methanol in the gel and the carbon dioxide surrounding the gel, the pressure was reduced to 4.0 $\mathrm{MPa}$ and then repressurized with carbon dioxide to 6.0 MPa. This procedure was repeated several times until the methanol was completely removed from the system. Subsequently, the autoclave was heated to $316 \mathrm{~K}$ which is above the critical temperature of carbon dioxide and kept there for at least one hour. After slow depressurization to atmospheric pressure, an opaque and brittle aerogel with

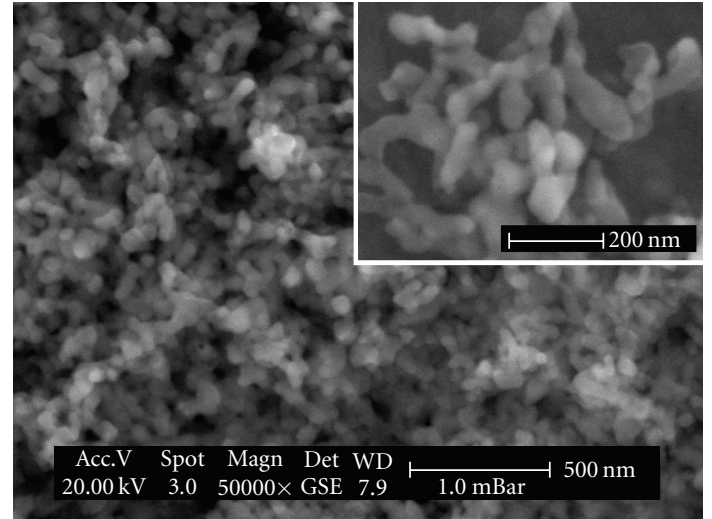

Figure 2: SEM pictures of the $\mathrm{CaCO}_{3}$ aerogel; magnifications: $50,000 \times$ (large picture) and 100,000 $\times$ (insert).

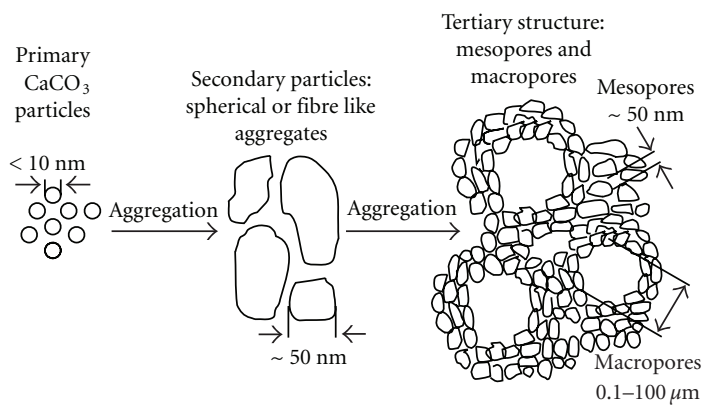

FIgURE 3: Schematic drawing of the reaction steps involved in the formation of the $\mathrm{CaCO}_{3}$ gel.

dimensions of $6 \times 2 \times 1 \mathrm{~cm}$ was obtained. The size of the aerogel was limited by the dimensions of the autoclave.

\section{Results and Discussion}

The sequence of reactions involved in the formation of the $\mathrm{CaCO}_{3}$ alcogel which is the precursor for the preparation of the $\mathrm{CaCO}_{3}$ aerogel is shown in Scheme 1. The aerogel was obtained by displacing methanol present in the alcogel with $\mathrm{CO}_{2}$.

The phase composition of the aerogel was determined by X-ray powder diffraction (Figure 1). The XRD pattern observed was identical with vaterite, a polymorph of calcium carbonate which is metastable at ambient temperature. The relatively large half width and the low intensity of the Bragg reflections indicate small particle sizes and only a moderate crystallinity.

A specific surface area (BET) of $45 \mathrm{~m}^{2} / \mathrm{g}$ was found for the calcium carbonate aerogel by nitrogen absorption. This value corresponds to an average particle diameter of approximately $50 \mathrm{~nm}$, provided the particles in the aerogel are discrete, monosized, and spherical.

In Figure 2, SEM pictures of the aerogel are shown. The secondary vaterite particles in the aerogel exhibit a spherical and/or fibre-like shape with an average diameter of approximately $50 \mathrm{~nm}$. This value corresponds quite well 
with the diameter calculated from the nitrogen adsorption measurement. As can be seen in the SEM pictures, the individual particles are not strongly connected with each other. In fact, they are merely aggregated, which explains the brittle character of the calcium carbonate aerogel when mechanical stress is applied.

The formation of the calcium carbonate aerogel occurs in a three-step process which is illustrated in Figure 3. First, calcium di(methylcarbonate) is hydrolyzed by water to form an intermediate sol containing primary $\mathrm{CaCO}_{3}$ nanoparticles showing a size of approximately 5 to $20 \mathrm{~nm}$. Existence of these primary particles was also confirmed by TEM pictures (not shown here). In a second step, the primary particles grow to spherical or fibre-like secondary particles which were observed under the SEM. In a third step, these secondary particles finally aggregate to the gel.

Because water is the starting reagent for seed formation in this system, the amount of water present during the reaction greatly influences the number, morphology, and size of the primary and secondary particles and therefore also the bulk properties of the aerogel.

\section{Conclusion}

Through the simple synthesis described here, calcium carbonate aerogels are readily available from inexpensive starting materials. Aerogels with different surface areas, specific densities, and pore sizes are accessible. Our process allows to produce monoliths with a volume of $20-30 \mathrm{~cm}^{3}$. Potential applications include heat insulating materials and fillers for plastics.

\section{References}

[1] S. S. Kistler, "Coherent expanded-aerogels," The Journal of Physical Chemistry, vol. 36, no. 1, pp. 52-64, 1932.

[2] S. S. Kistler, "Coherent expanded aerogels and jellies," Nature, vol. 127, no. 3211, p. 741, 1931.

[3] S. S. Kistler, "The relation between heat conductivity and structure in silica aerogel," The Journal of Physical Chemistry, vol. 39, no. 1, pp. 79-86, 1935.

[4] S. S. Kistler, E. A. Fischer, and I. R. Freeman, "Sorption and surface area in silica aerogel," Journal of the American Chemical Society, vol. 65, no. 10, pp. 1909-1919, 1943.

[5] M. Schmidt and F. Schwertfeger, "Applications for silica aerogel products," Journal of Non-Crystalline Solids, vol. 225, no. 1, pp. 364-368, 1998.

[6] J. Fricke and A. Emmerling, "Aerogels," Journal of the American Ceramic Society, vol. 75, no. 8, pp. 2027-2035, 1992.

[7] Yu. K. Akimov, "Fields of application of aerogels (review)," Instruments and Experimental Techniques, vol. 46, no. 3, pp. 287-299, 2003.

[8] R. W. Pekala, "Organic aerogels from the polycondensation of resorcinol with formaldehyde," Journal of Materials Science, vol. 24, no. 9, pp. 3221-3227, 1989.

[9] C. Hoang-Van, B. Pommier, R. Harivololona, and P. Pichat, "Alumina-based aerogels as carriers for automotive palladium catalysts," Journal of Non-Crystalline Solids, vol. 145, pp. 250 254, 1992.
[10] B. E. Yoldas, "Alumina gels that form porous transparent $\mathrm{Al}_{2} \mathrm{O}_{3}$," Journal of Materials Science, vol. 10, no. 11, pp. 18561860, 1975.

[11] J. Livage, M. Henry, and C. Sanchez, "Sol-gel chemistry of transition metal oxides," Progress in Solid State Chemistry, vol. 18, no. 4, pp. 259-341, 1988.

[12] A. E. Gash, T. M. Tillotson, J. H. Satcher Jr., L. W. Hrubesh, and R. L. Simpson, "New sol-gel synthetic route to transition and main-group metal oxide aerogels using inorganic salt precursors," Journal of Non-Crystalline Solids, vol. 285, no. 13, pp. 22-28, 2001.

[13] R. Horga, F. Di Renzo, and F. Quignard, "Ionotropic alginate aerogels as precursors of dispersed oxide phases," Applied Catalysis A, vol. 325, no. 2, pp. 251-255, 2007.

[14] A. Buzagh, "On colloidal solutions of alkaline earth carbonates," Kolloid-Zeitschrift, vol. 38, no. 3, pp. 222-226, 1926. 

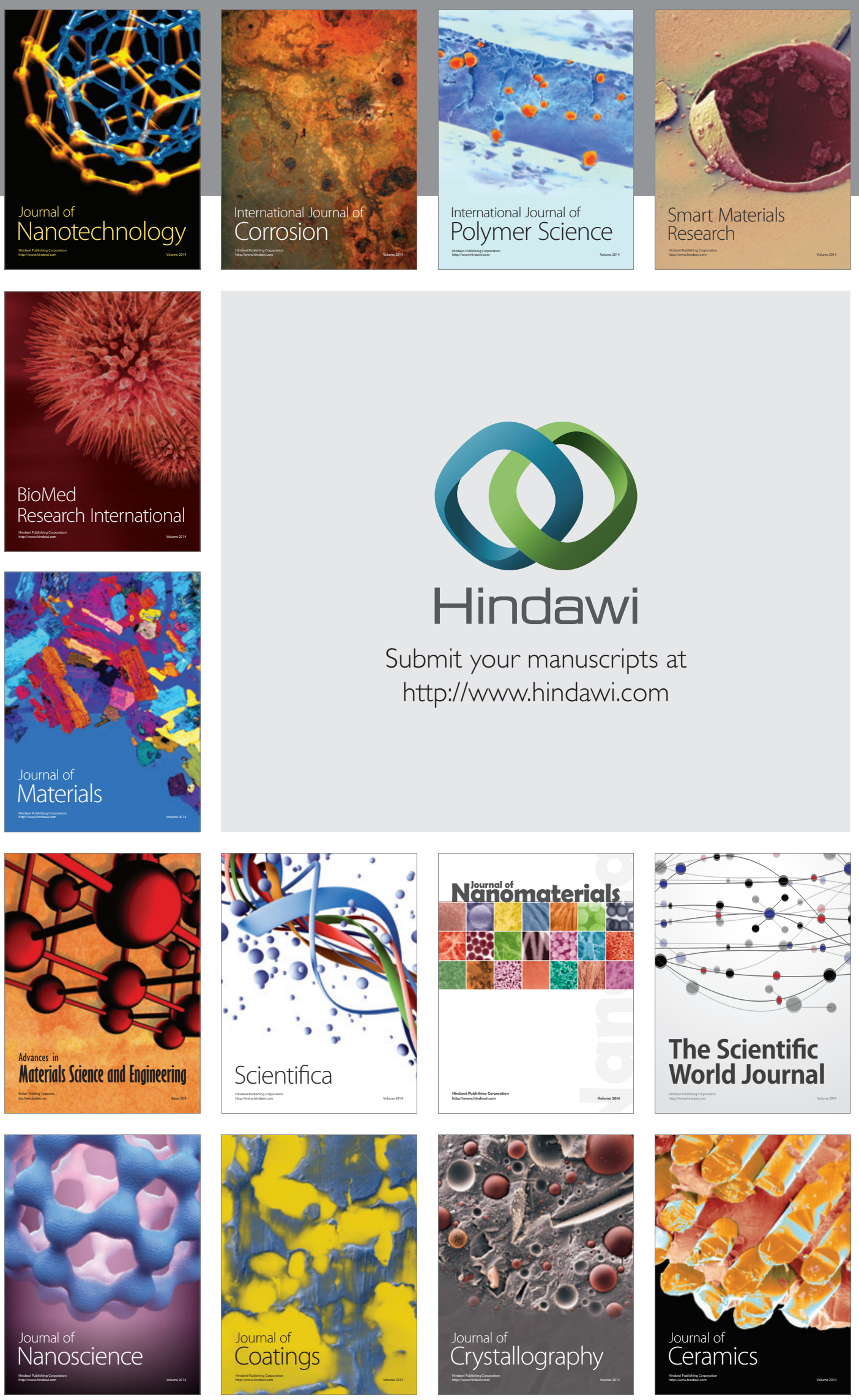

The Scientific World Journal

Submit your manuscripts at

http://www.hindawi.com

\section{World Journal}

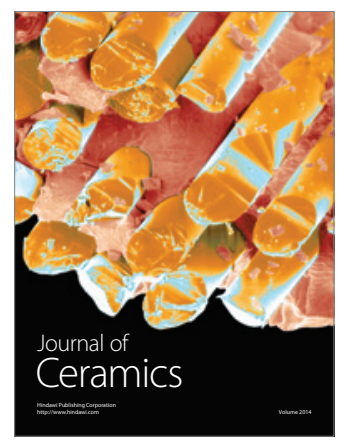

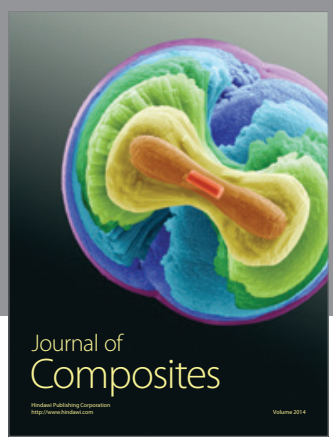
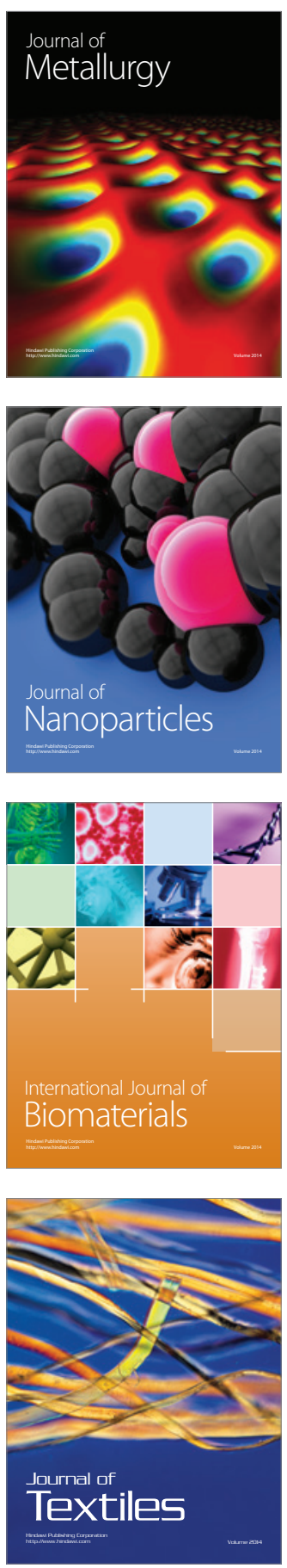